\title{
Time Reversal Mirror and Perfect Inverse Filter in a Microscopic Model for Sound Propagation
}

\author{
Hernán L. Calvo, Ernesto P. Danieli, Horacio M. Pastawski*, \\ Facultad de Matemática, Astronomía y Física, Universidad Nacional de Córdoba, Ciudad Universitaria, 5000 Córdoba, \\ Argentina
}

\begin{abstract}
Time reversal of quantum dynamics can be achieved by a global change of the Hamiltonian sign (a hasty Loschmidt daemon), as in the Loschmidt Echo experiments in NMR, or by a local but persistent procedure (a stubborn daemon) as in the Time Reversal Mirror (TRM) used in ultrasound acoustics. While the first is limited by chaos and disorder, the last procedure seems to benefit from it. As a first step to quantify such stability we develop a procedure, the Perfect Inverse Filter (PIF), that accounts for memory effects, and we apply it to a system of coupled oscillators. In order to ensure a many-body dynamics numerically intrinsically reversible, we develop an algorithm, the pair partitioning, based on the Trotter strategy used for quantum dynamics. We analyze situations where the PIF gives substantial improvements over the TRM.
\end{abstract}

In the last years, the group of M. Fink in Paris developed an experimental technique called Time Reversal Mirror (TRM) that allows the time reversal of acoustic excitations [1]. An ultrasonic pulse, produced inside a control region (also called cavity) where it suffers multiple scattering processes, is detected by several microphones as it escapes through the boundaries. These transducers can also act as loudspeakers and the registered signal is played back in the time reversed sequence. Thus, the signal focalizes in the source point forming a Loschmidt Echo [2]. According to the existing theory, an exact control of the wave function in the cavity would require the control of the wave function and the normal derivative at the boundaries. However, the reversal is quite good even when these conditions are not fulfilled by the experiment: the detectors might not enclose the cavity or the recording time period could be reduced to a fraction. Another surprising feature of this time reversion procedure is that it shows a much better stability in inhomogeneus media as compared to ordered ones. This leads to numerous appli-

\footnotetext{
* Corresponding author.

Email address: horacio@famaf.unc.edu.ar (Horacio M. Pastawski).
}

cations in medical physics [3] and communications [4]. A first step to asses the errors is to develop a procedure that could achieve perfect reversal. This task was developed for the domain of quantum waves and was named Perfect Inverse Filter (PIF) [5]. The PIF procedure assures the exact reversion by injecting a wave function that compensates precisely the feedback effects through a frequency dependent renormalization that involves the exact Green's function at the injection sites. Here, we use a simple microscopic model that presents wave behavior and describes energy dissipation to show that the PIF procedure also applies in the classical domain. The model, represented in Fig. 1, is a variation of that of Rubin [6]: a surface oscillator with mass $m_{0}$ and natural frequency $\omega_{0}$ is coupled to a semi-infinite harmonic chain of bulk oscillators with mass $m$. We are interested in the time reversion of the initial condition where all the oscillators are in their equilibrium positions except for the surface one. The energy stored in the surface oscillator is expected to decay due to the effective friction produced by the "environment" of light masses. The Hamiltonian is: 


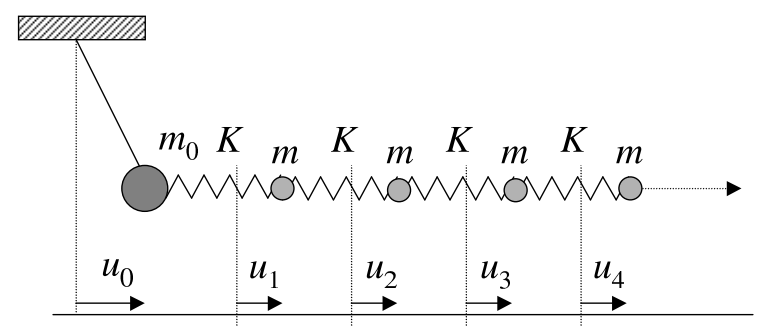

Fig. 1. Scheme of the model: a simple pendulum (surface oscillator) is coupled to the bulk masses.

$H=\sum_{i}\left(\frac{p_{i}^{2}}{2 m_{i}}+\frac{m_{i} \omega_{i}^{2}}{2} u_{i}^{2}\right)+\sum_{i} \frac{K_{i, i+1}}{2}\left(u_{i}-u_{i+1}\right)^{2}$,

where $\omega_{i}$ is the natural frequency of the $i$ th oscillator and $u_{i}$ describes the displacement from the equilibrium. Springs with elastic constant $K_{i, i+1}$ accounts for the coupling between first neighbors. For the proposed model, all springs and masses are equal and $\omega_{i}=0$, except for the heavier surface mass that suffers an additional harmonic restitutive force ( $\omega_{0}$ is finite). The exchange frequency $\omega_{\mathrm{x}}=\sqrt{K / m}$ and the ratio $\alpha=$ $m / m_{0}<1$ have been chosen to set the system in the extended-oscillatory dynamical regime [7]. The equations of motion in the frequency domain can be written in the matrix form

$$
\mathbb{D}^{-1}(\omega) \mathbf{u}(\omega)=\left(\omega^{2} \mathbb{I}-\mathbb{M}\right) \mathbf{u}(\omega)=0,
$$

where $\mathbb{D}(\omega)=\left(\omega^{2} \mathbb{I}-\mathbb{M}\right)^{-1}$ is the resolvent associated to the dynamical matrix in the site basis

$$
\mathbb{M}=\left(\begin{array}{cccc}
\omega_{0}^{2}+\alpha \omega_{\mathrm{x}}^{2} & -\alpha \omega_{\mathrm{x}}^{2} & 0 & \cdots \\
-\omega_{\mathrm{x}}^{2} & 2 \omega_{\mathrm{x}}^{2} & -\omega_{\mathrm{x}}^{2} & \\
0 & -\omega_{\mathrm{x}}^{2} & 2 \omega_{\mathrm{x}}^{2} & \\
\vdots & & & \ddots
\end{array}\right)
$$

The resolvent provides the solutions to Eq. 2 with impulsive forces $f_{i}(t)=m_{i} \Delta \dot{u}_{i}(0) \delta(t)$. This is,

$$
\begin{aligned}
u_{j}(t) & =\sum_{i} \int \frac{\mathrm{d} \omega}{2 \pi} e^{-\mathrm{i} \omega t} D_{j, i}(\omega) \Delta \dot{u}_{i}(0) \\
& =\sum_{i} D_{j, i}(t) \Delta \dot{u}_{i}(0)
\end{aligned}
$$

Notice that $D_{j, i}(t)$ relates the $j$ th displacement amplitude due to an initial condition of velocity in the $i$ th oscillator. In general, the solution of the Eq. 2 in presence of forces $F_{i}(t)$ results

$$
u_{j}(t)=\sum_{i} \int_{0}^{t} \chi_{j, i}\left(t-t^{\prime}\right) F_{i}\left(t^{\prime}\right) \mathrm{d} t^{\prime},
$$

and can be rewritten as

$$
u_{j}(\omega)=\sum_{i} \chi_{j, i}(\omega) F_{i}(\omega)=\sum_{i} D_{j, i}(\omega)\left(\frac{-1}{m_{i}}\right) F_{i}(\omega)
$$

where $F_{i}(\omega)=f_{i}(\omega)+g_{i}(\omega)$ is the Fourier transform of the force applied at mass $i$ that is a sum of two components: an impulsive force and a shifting force. This last is able to produce an "instantaneous" shift $\Delta u_{j}(0)$ in the position without changing its momentum. This would require that a first "impulsive kick" be followed by a compensating one:

$$
g_{i}(t)=\lim _{\tau \rightarrow 0} m_{i} \Delta u_{i}(0) \frac{1}{\tau}\left[\delta\left(t+\frac{1}{2} \tau\right)-\delta\left(t-\frac{1}{2} \tau\right)\right],
$$

which in frequency domain means:

$$
g_{i}(\omega)=-\mathrm{i} \omega m_{i} \Delta u_{i}(0) .
$$

Thus, in the time domain

$$
\begin{aligned}
u_{j}(t) & =\sum_{i} \int \frac{\mathrm{d} \omega}{2 \pi}(-\mathrm{i} \omega) \chi_{j, i}(\omega) e^{-\mathrm{i} \omega\left(t-t^{\prime}\right)} m_{i} \Delta u_{i}\left(t^{\prime}\right) \\
& =\sum_{i} G_{j, i}\left(t-t^{\prime}\right) \Delta u_{i}\left(t^{\prime}\right),
\end{aligned}
$$

which serves as a definition for the position-position response function, also called the Green's function:

$$
G_{j, i}(\omega)=-\mathrm{i} \omega D_{j, i}(\omega)=\mathrm{i} \omega \chi_{j, i}(\omega) m_{i} .
$$

On the other hand we will use linearity to write the observed displacement in terms of $\delta u_{i}(t)$, the forced position shift accumulated in the unit time:

$$
u_{j}(t)=\sum_{i} \int G_{j, i}\left(t-t^{\prime}\right) \delta u_{i}\left(t^{\prime}\right) \mathrm{d} t^{\prime} .
$$

We seek the injection function $\delta u_{i}(t)$ that produces the exact reversion of the original wave within the control region, i.e. $u_{j}^{\text {rev }}(t) \equiv u_{j}^{*}\left(2 t_{R}-t\right)$ for $t_{R} \leq t \leq 2 t_{R}$. According to Ref. [5], the perfect time reversal is possible if the dynamics starts and ends up without any excitation inside the cavity. Even when our system starts with an "excited" cavity, the lack of momentum at each mass ensures that forward and backwards evolutions are identical. Once the decay signal is registered at the transducer for positive time, the earlier time values (corresponding to a fictitious injection) are also known and we build the function to be inverted at time $t_{R}$

$$
\tilde{u}_{s}(t)=\left\{\begin{array}{l}
u_{s}^{*}\left(t_{R}-t\right), \quad-t_{R} \leq t \leq 0 \\
u_{s}\left(t-t_{R}\right), \quad 0 \leq t \leq t_{R}
\end{array},\right.
$$

and the injection function can be obtained in the frequency domain by

$$
\delta u_{s}(\omega)=\frac{\tilde{u}_{s}^{*}(\omega)}{G_{s, s}(\omega)} .
$$

This equation defines the Perfect Inverse Filter (PIF) for a classical wave, where the injection prescribed by the TRM procedure appears now corrected by the Green's function $G_{s, s}(t)$. The time evolution of the displacement amplitude is shown in Fig. 2 for several situations. The recording time $t_{R}$ is longer than the decay 


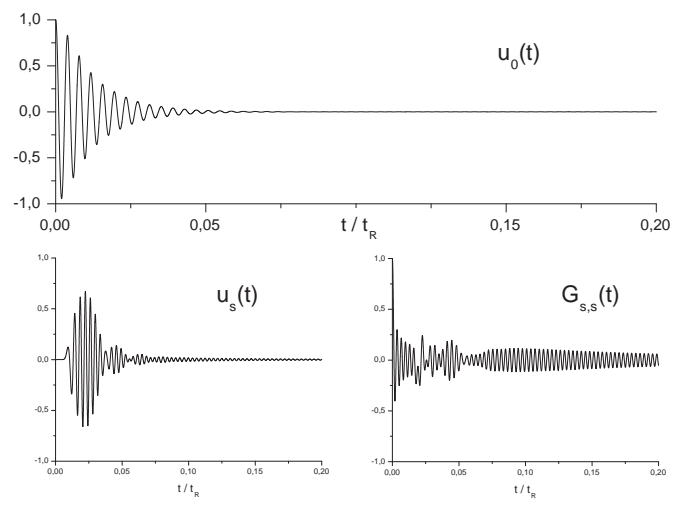

Fig. 2. Displacement amplitudes. Up: initial condition at the surface oscillator. Down - left: registered signal in $x_{s}$. Down - right: response function in $x_{s}$. The chosen parameters are: $\alpha=0.25, \omega_{0}=1.5 \omega_{\mathrm{x}}, t_{R}=1000 \omega_{\mathrm{x}}^{-1}$ and $x_{s}=10$.

time in the whole cavity, i.e. all the masses in the control region have enough time to recover their equilibrium positions. The injection functions of both procedures differs near the bandedges $\left(\omega= \pm 2 \omega_{\mathrm{x}}\right)$ indicating that TRM corrections are importants in cases where the whole spectrum is involved (typically in broadband experiments). When all the masses in the cavity are in their equilibrium position, the injection at the source point $x_{s}$ produces oscillations that propagate both sides of $x_{s}$. Only dynamics inside the cavity is fully reversed. Fig. 3 shows the local energy of the surface oscillator

$$
E_{0}(t)=\frac{1}{2} m_{0}\left|\dot{u}_{0}(t)\right|^{2}+\frac{1}{2} m_{0}\left(\omega_{0}^{2}+\alpha \omega_{\mathrm{x}}^{2}\right)\left|u_{0}(t)\right|^{2} ;
$$

its decay and recovering presents the three temporal domains [8]: It begins with a quadratic dependence, continues with an exponential decay associated with a Self Consistent Fermi Golden Rule, and becomes an inverse power law when the energy return is comparable to the residual energy in the surface mode. The time reversed signal reproduces all the three regimes, even when they involve very different signal intensities. We attempt to asses the quality of the reversal by the Loschmidt Echo [2] of a wave function $\varphi(t)$ normalized in the cavity:

$$
M(t)=\left|\left\langle\varphi_{\mathrm{rev}}(t) \mid \varphi\left(2 t_{R}-t\right)\right\rangle\right|^{2} \quad t_{R} \leq t \leq 2 t_{R}
$$

First, the inner product uses a metric tensor given by the Hamiltonian, i.e. we refer the recovered energy to that originally contained within the control region. Considering the case where $t_{R}=1000 \omega_{\mathrm{x}}^{-1}$, the PIF yields $M_{\mathrm{PIF}}=0.999$ while the TRM gives $M_{\mathrm{TRM}}=$ 0.982 . This result is not representative of the evidenced by Fig. 4. Alternatively, we use the Euclidean metric tensor, obtaining $M_{\mathrm{PIF}}=1$ and $M_{\mathrm{TRM}}=0.765$, i.e. in PIF all the initial condition have been recov-

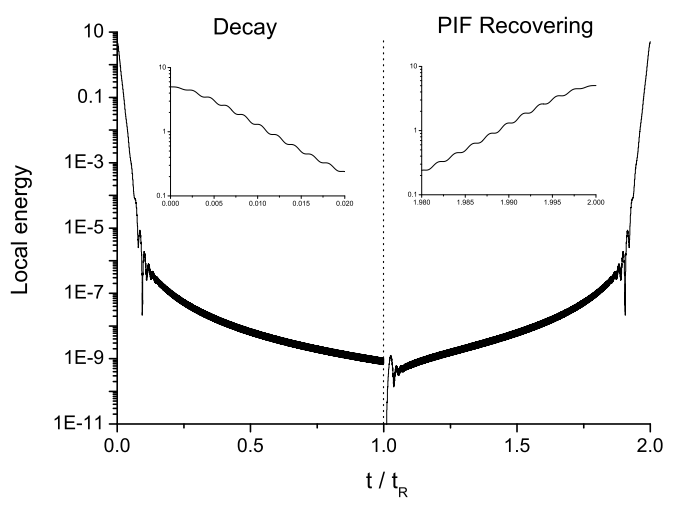

Fig. 3. Decay and recovering by PIF procedure of the local energy in logarithmic scale. The insets show in detail the quadratic and exponential regimes.

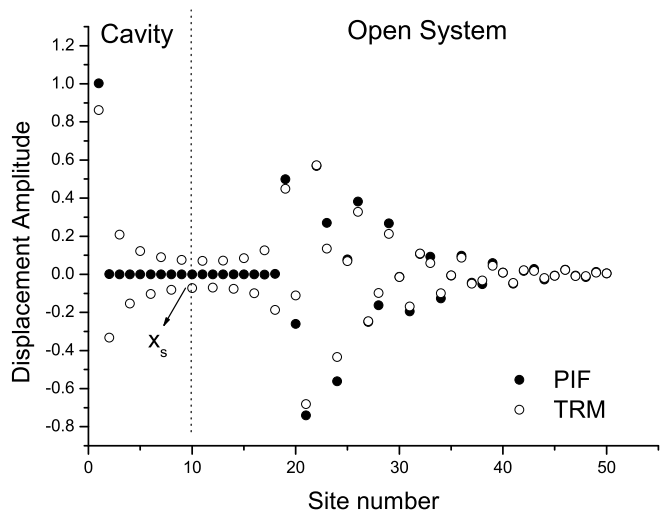

Fig. 4. Recovering of the initial condition for the two procedures.

ered whereas in TRM there is a spreading of displacements and velocities along the cavity. The reversed local energy in both procedures is compared in Fig. 5. The PIF procedure can not be distinguished from the ideal reversal. The TRM has two failures: the amplitude of the local energy at $t=2 t_{R}$ is always less than the initial case and the temporal regimes are delayed. In summary, the time reversion of the dynamics of a pendulum coupled to an harmonic chain has been done by means of two types of "stubborn daemons": 1) The TRM, which neglects memory effects (meaning that an instantaneous system response is assummed). 2) The PIF, that accounts for memory and feedback. This last is much better if the initial condition is build up over the whole range of frequencies. In this case, the correction of the Green's function becomes non trivial and ensures a better reversion quality. 


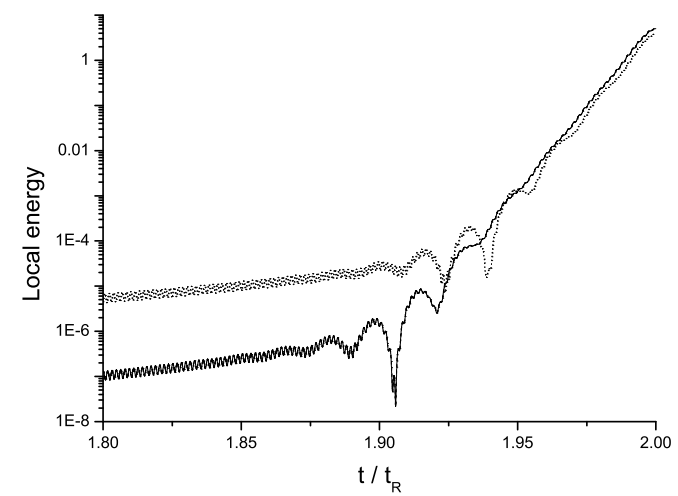

Fig. 5. Focalization of the local energy in logaritmic scale. Solid line represents the ideal time reversed decay, dashed line is PIF and TRM is shown in dotted line. Note that PIF is superposed with the ideal case.

\section{Appendix A. Classical dynamics through pair partitioning}

While the 1D dynamics in the model considered can be obtained analytically by the continued fraction method [9], we also evaluate it numerically by developing an algorithm, the pair partitioning, inspired in the Trotter strategy used in quantum dynamics [10]. We split the kinetic terms to rewrite the Hamiltonian as

$$
H=\sum_{i} H_{i, i+1} \text {. }
$$

Now, each term represents an effective Hamiltonian for two coupled oscillators, with twice the mass and half of the natural frequency each. Pair dynamics is solved analytically and impose a periodic evolution sequence that alternates each coupled pair according to their parity. The total energy is not exactly conserved but fluctuates with an amplitude $\Delta E$ around the ideal conserved value. Since $\Delta E$ is proportional to $\delta t^{2}$, the square of the temporal step, it becomes negligible for typical cases where $\delta t=0.01 \omega_{\mathrm{x}}^{-1}$. The fact that each piecelike dynamics is perfectly reversible is very important for the test of different time reversal procedures.

\section{References}

[1] M. Fink, Sci. Am. 281, May issue (1999) 67.

[2] R. Jalabert and H.M. Pastawski, Phys. Rev. Lett. 86 (2001) 2490

[3] M. Fink, G. Montaldo, M. Tanter, Annual Rev. Biomed. Eng. 5 (2003) 465.

[4] G.F. Edelman, T. Akal, W.S. Hodkiss, S. Kim, W.A. Kuperman, H.C. Song, IEEE J. Ocean Eng. 27 (2002) 602.
[5] H.M. Pastawski, E.P. Danieli, H.L. Calvo, L.E.F. Foa Torres, Europhys. Lett. 77 (2007) 40001.

[6] R.J. Rubin, Phys. Rev. 1313 (1963) 964.

[7] H.L. Calvo, H.M. Pastawski, Braz. J. Phys. 36 3B (2006) 963.

[8] E. Rufeil Fiori and H.M. Pastawski, Chem. Phys. Lett. 420 (2006) 35.

[9] H.M. Pastawski and E. Medina, Rev. Mex. Física 47s1 (2001) 1, cond-mat/0103219

[10] H. De Raedt, Ann. Rev. of Comp. Physics IV (1996) 107. 